International Journal of Modern Physics C,

(C) World Scientific Publishing Company

\title{
CALCULATION OF PERCOLATION THRESHOLDS IN HIGH DIMENSIONS FOR FCC, BCC AND DIAMOND LATTICES
}

\author{
Steven C. van der Marck \\ SIEP Research and Technical Services, P.O. Box 60 \\ 2280 AB Rijswijk, The Netherlands. \\ e-mail: s.c.vandermarck@siep.shell.com
}

Received March 24, 1998

Revised

\begin{abstract}
Site and bond percolation thresholds are calculated for the face centered cubic, body centered cubic and diamond lattices in 4,5 , and 6 dimensions. The results are used to study the behaviour of percolation thresholds as a functions of dimension. It is shown that the predictions from a recently proposed invariant for percolation thresholds are not satisfactory for these lattices.
\end{abstract}

Keywords: percolation threshold, high dimension, lattice, fcc, bcc, diamond

\section{Introduction}

Percolation problems have a wide range of applicability, and have therefore attracted a fair bit of attention over many years]. Nevertheless the percolation thresholds, which are among the basic quantities for percolation on lattices, have been calculated exactly for only a few two-dimensional lattices. For many other lattices these thresholds have been calculated numerically. These numerical values can then be analysed, to determine regular behaviour, or trends, as a function of the lattice coordination number, dimensionality, etc. A limited review of such efforts has been given elsewherel.

Recently, Galam and Mauger 3 proposed an invariant for site and bond percolation thresholds, $p_{c s}$ and $p_{c b}$ respectively. The proposed invariant reads

$$
\left(p_{c s}\right)^{1 / a_{s}}\left(p_{c b}\right)^{-1 / a_{b}}=\frac{\delta}{d},
$$

where $d$ is the dimension of the lattice, and $a_{s}, a_{b}$, and $\delta$ are positive constants. The lattices studied by Galam and Mauger were divided into two classes, and for each of these classes the values of the parameters were fitted. The values for the first class were $\left\{a_{s}=0.3670 ; a_{b}=0.6897 ; \delta=1.3638\right\}$, while for the second class $\left\{a_{s}=0.6068 ; a_{b}=0.9346 ; \delta=1.9340\right\}$. For the lattices used by Galam and Mauger, the numerical results for this invariant are indeed constant within 5\%. Although the deviations up to $5 \%$ cannot be explained by the inaccuracy in the values for the percolation thresholds, it is an interesting observation that the above combination 
Table 1. The site percolation thresholds of $d$-dimensional fcc lattices as a function of linear lattice size $L$. The values in the last row are results of a fit to the scaling relation $\left|p_{c}(L)-p_{c}(\infty)\right| \propto L^{-1 / \nu}$. Error estimates concerning the last digit are indicated between brackets.

\begin{tabular}{rcrcrlrc}
\hline \multicolumn{2}{c}{$d=3$} & & $d=4$ & & $d=5$ & \multicolumn{2}{c}{$d=6$} \\
$L$ & $p_{c s}$ & $L$ & $p_{c s}$ & $L$ & $p_{c s}$ & $L$ & $p_{c s}$ \\
\hline 16 & $0.2097(2)$ & 16 & $0.0856(2)$ & 8 & $0.0457(2)$ & 8 & $0.0257(2)$ \\
32 & $0.2035(2)$ & 24 & $0.0847(2)$ & 12 & $0.0435(2)$ & 10 & $0.0258(2)$ \\
64 & $0.2016(2)$ & 32 & $0.0845(2)$ & 16 & $0.0432(2)$ & 12 & $0.0252(2)$ \\
128 & $0.2001(2)$ & 48 & $0.0844(2)$ & 24 & $0.0431(2)$ & 14 & $0.0252(2)$ \\
250 & $0.1998(2)$ & 64 & $0.0843(2)$ & 32 & $0.0432(2)$ & 16 & $0.0252(2)$ \\
$\infty$ & $0.1994(2)$ & $\infty$ & $0.0842(3)$ & $\infty$ & $0.0431(3)$ & $\infty$ & $0.0252(5)$ \\
\hline
\end{tabular}

of percolation thresholds yields almost constant values. Especially the absence of coordination number $q$ in the invariant makes it valuable.

However, the invariant can be tested on more lattices for which the percolation thresholds are known. We list data for many lattices in this article, obtained from various sources in literature. However, for some of the higher dimensional lattices, only site- or only bond percolation thresholds were known. Therefore the 'missing' values are computed here, so that the invariant can be calculated for these lattices as well. Moreover, it is interesting to study the scaling of percolation thresholds as a function of dimension for some of the important lattices, like the face centered cubic lattice (fcc), the body centered cubic lattice (bcc) and the diamond lattice.

This paper is organised as follows. In section 2, definitions are given for the fcc, bcc, and diamond lattices in higher dimensions. The cluster algorithm and some implementation issues are discussed in section 3, after which the results are presented in section 4 . Finally, section 5 contains a discussion of the results.

\section{Lattice definitions in higher dimensions}

For the definitions of lattices in $d$ dimensions, we refer to Conway and Sloand for the face centered cubic and body centered cubic lattice, and to Van der Marck for the diamond and Kagomé lattice. For completeness, we briefly describe here the generalisation of the fcc, bcc and diamond lattices to higher dimensions. First we need to have, for each lattice, a set of $d$ independent lattice vectors. A point is called a lattice site if and only if it is an integer combination of these vectors. Secondly, we want to know the neighbours of a site, described in terms of the lattice vectors. We will denote an orthonormal basis of $d$-dimensional space by $\mathbf{x}_{i}$.

The $d$-dimensional fcc lattice is the set of points in $\mathbb{Z}^{n}$, for which the sum of the coordinates is even. Conway and Sloane use the notation ' $D_{d}$ ' for this lattice $\mathbb{E}$. (Note that $D_{d}$ is the 'closest packed' lattice for $d=3,4$ and 5, but not for higher dimensions $\mathbf{l}$.) Each site has $2 d(d-1)$ neighbours at a relative location $\pm \mathbf{x}_{i} \pm \mathbf{x}_{j}$, for $i, j=1, \ldots, d(i \neq j)$. As a set of lattice vectors one can choose $\mathbf{f}_{i}=\mathbf{x}_{1}+\mathbf{x}_{i}$. The neighbours of a site are given in terms of these lattice vectors as

$$
\left.\begin{array}{l} 
\pm\left(\mathbf{f}_{i}-\mathbf{f}_{j}\right) \\
\pm\left(\mathbf{f}_{i}+\mathbf{f}_{j}-\mathbf{f}_{1}\right)
\end{array}\right\} \text { for all } i, j=1, \ldots, d \quad(i \neq j) .
$$


Table 2. The site percolation thresholds of $d$-dimensional bcc lattices as a function of linear lattice size $L$. The values in the last row are results of a fit to the scaling relation $\left|p_{c}(L)-p_{c}(\infty)\right| \propto L^{-1 / \nu}$. Error estimates concerning the last digit are indicated between brackets.

\begin{tabular}{rcrcrlrc}
\hline \multicolumn{2}{c}{$d=3$} & \multicolumn{2}{c}{$d=4$} & \multicolumn{2}{c}{$d=5$} & \multicolumn{2}{c}{$d=6$} \\
$L$ & $p_{c s}$ & $L$ & $p_{c s}$ & $L$ & $p_{c s}$ & \multicolumn{1}{c}{$L$} & $p_{c s}$ \\
\hline 16 & $0.2593(2)$ & 16 & $0.1078(2)$ & 8 & $0.0497(2)$ & 8 & $0.0215(2)$ \\
32 & $0.2514(2)$ & 24 & $0.1058(2)$ & 12 & $0.0468(2)$ & 10 & $0.0205(2)$ \\
64 & $0.2483(2)$ & 32 & $0.1050(2)$ & 16 & $0.0459(2)$ & 12 & $0.0203(2)$ \\
128 & $0.2471(2)$ & 48 & $0.1043(2)$ & 24 & $0.0453(2)$ & 14 & $0.0201(2)$ \\
250 & $0.2463(2)$ & 64 & $0.1042(2)$ & 32 & $0.0450(2)$ & 16 & $0.0202(2)$ \\
$\infty$ & $0.2458(2)$ & $\infty$ & $0.1037(3)$ & $\infty$ & $0.0446(4)$ & $\infty$ & $0.0199(5)$ \\
\hline
\end{tabular}

A site in the $d$-dimensional bcc lattice has $2^{d}$ neighbours, located at $\frac{1}{2}\left( \pm \mathbf{x}_{1} \pm\right.$ $\left.\mathbf{x}_{2} \pm \ldots \pm \mathbf{x}_{d}\right)$. This is dubbed the 'generalized bcc net' by Conway and Sloane $\mathbf{A}$. A possible set of lattice vectors is $\mathbf{b}_{i}=-\frac{1}{2}\left(\mathbf{x}_{1}+\ldots+\mathbf{x}_{i-1}\right)+\frac{1}{2}\left(\mathbf{x}_{i}+\ldots+\mathbf{x}_{d}\right)$ for $i=1, \ldots, d$. The neighbours of a site are given in terms of these vectors by

$$
\begin{array}{ll} 
\pm \mathbf{b}_{i} & \text { for } i=1, \ldots, d \\
\pm\left(\mathbf{b}_{i}-\mathbf{b}_{j}+\mathbf{b}_{k}\right) & \text { for } i>j>k \\
\pm\left(\mathbf{b}_{i}-\mathbf{b}_{j}+\mathbf{b}_{k}-\mathbf{b}_{l}+\mathbf{b}_{m}\right) & \text { for } i>j>k>l>m \\
\ldots &
\end{array}
$$

The $d$-dimensional diamond lattice is a lattice with a 2-point basis. Let us call the two points in the basis A and B. The full lattice is built by translation of the lattice basis over $d$ independent vectors $\mathbf{t}_{i}$. Each A-site has $d+1$ neighbours of type B. One of these neighbours is the B-site in the same basis, while the other $d$ neighbours are the B-type sites at a relative location $\mathbf{t}_{i}$. Each B-site also has $d+1$ neighbours, one of which is the A-site in the same basis and the others are the A-type sites at a relative location $-\mathbf{t}_{i}$.

\section{Lattice coding and cluster algorithm}

Two programs to calculate percolation thresholds were developed. One program was geared towards the handling of any desired lattice topology, the other towards speed and efficient memory usage for certain specific lattices ${ }^{\mathrm{a}}$.

In the general purpose program to calculate percolation thresholds we used a generic method to specify a lattice. The bonds connected to lattice sites were coded in Fortran as NB_SITES (QMAX, NSITES), where NSITES is the total number of sites in the lattice, and QMAX is the maximum number of bonds connected to a site (maximum coordination number). The integer value of NB_SITES (I,S) was set to the bond number of the $i^{\text {th }}$ bond connected to site $s$.

The sites connected to bonds were coded as NB_BONDS ( 2 , NBONDS), where NBONDS is the total number of bonds in the lattice. In other words, link $i$ connects site NB_BONDS $(1, I)$ and NB_BONDS $(2, I)$. The two arrays NB_SITES and NB_BONDS together specify the topology of the lattice completely.

aThe programs, one in Fortran and one in $\mathrm{C}$, can be obtained from the author. 
Table 3. The site percolation thresholds of $d$-dimensional diamond lattices as a function of linear lattice size $L$. The values in the last row are results of a fit to the scaling relation $\left|p_{c}(L)-p_{c}(\infty)\right| \propto$ $L^{-1 / \nu}$. Error estimates concerning the last digit are indicated between brackets.

\begin{tabular}{rcrcrcrcrc}
\hline \multicolumn{2}{c}{$d=2$} & \multicolumn{2}{c}{$d=3$} & \multicolumn{2}{c}{$d=4$} & \multicolumn{2}{c}{$d=5$} & \multicolumn{2}{c}{$d=6$} \\
$L$ & $p_{c s}$ & $L$ & $p_{c s}$ & $L$ & $p_{c s}$ & $L$ & $p_{c s}$ & $L$ & $p_{c s}$ \\
\hline 256 & $0.6964(2)$ & 16 & $0.4398(2)$ & 16 & $0.3014(2)$ & 8 & $0.2268(2)$ & 8 & $0.1760(2)$ \\
512 & $0.6964(2)$ & 32 & $0.4347(2)$ & 24 & $0.2996(2)$ & 12 & $0.2251(2)$ & 10 & $0.1762(2)$ \\
1024 & $0.6969(2)$ & 64 & $0.4317(2)$ & 32 & $0.2989(2)$ & 16 & $0.2248(2)$ & 12 & $0.1770(2)$ \\
2048 & $0.6970(2)$ & 128 & $0.4312(2)$ & 50 & $0.2984(2)$ & 24 & $0.2249(2)$ & 14 & $0.1777(2)$ \\
3072 & $0.6970(1)$ & 250 & $0.4306(2)$ & 64 & $0.2983(2)$ & 30 & $0.2251(2)$ & 16 & $0.1783(2)$ \\
$\infty$ & $0.6971(2)$ & $\infty$ & $0.4301(2)$ & $\infty$ & $0.2978(2)$ & $\infty$ & $0.2252(3)$ & $\infty$ & $0.1799(5)$ \\
\hline
\end{tabular}

When we want to calculate a percolation threshold for such a lattice, we need a cluster algorithm, e.g. the one proposed by Hoshen and Kopelmant. This algorithm works by assigning a cluster label $m$ to each site and bond. (It is assumed that $m>0$.) In addition to the arrays to hold these labels, the algorithm uses one other array $N(m)$. For each proper label, $N(m)$ is greater than zero (by definition of 'proper'), in which case it holds the number of sites and bonds that belong to the cluster $m$. For a non-proper label $N(m)$ is negative, and $-N(m)$ refers to a label $m^{\prime}$, which is the cluster that cluster $m$ has been merged with.

The algorithm consists of the following steps.

- Initialise by setting $N(m) \leftarrow 0$ for all $m$, and $m_{\text {next }} \leftarrow 1$.

- Loop over all sites and bonds of the lattice.

- For each new occupied site and bond one encounters, one has to determine which cluster it belongs to. To this end, loop over the neighbours that one already determined a cluster label for. Three different situations can occur.

$=\mathbf{0}$ If there are no labeled neighbours, one has to define a new cluster label.

Use $m \leftarrow m_{\text {next }}$, set $N(m) \leftarrow 1$, and $m_{\text {next }} \leftarrow m_{\text {next }}+1$.

$=\mathbf{1}$ If there is one labeled cluster $m$, the current site/bond will get the label of that cluster. Set $N(m) \leftarrow N(m)+1$.

$\geq 2$ If there are two or more neighbours that belong to different clusters, then those clusters have to be 'merged':

- Determine the lowest cluster label, $m_{l o w}$, of these neighbours.

- For all other labels $m_{\text {other }}$, set $N\left(m_{\text {other }}\right) \leftarrow-m_{\text {low }}$, and $N\left(m_{\text {low }}\right) \leftarrow N\left(m_{\text {low }}\right)+N\left(m_{\text {other }}\right)$.

- For the current site or bond, set $m \leftarrow m_{\text {low }}$, and $N(m) \leftarrow N(m)+1$.

In this algorithm, it is important to know the neighbours of a site or bond. The specification of neighbours is the only item that is lattice-specific. When one is interested in site-bond percolation, the neighbours of a site are directly given by the NB_SITES array, and the neighbours of a bond by the NB_BONDS array. Therefore 
the implementation of the Hoshen-Kopelman algorithm is fairly straightforward for site-bond problems. There is but one important difference with the algorithm as described by Hoshen and Kopelman, which is that in their article, the networks could be traversed in an ordered way, whereas here we cannot. Let us take as an example the square lattice in two dimensions. If the loop over sites is performed as



it is clear that neighbouring sites $\{i, j-1\}$ and $\{i-1, j\}$ have been visited before site $\{i, j\}$, whereas $\{i+1, j\}$ and $\{i, j+1\}$ have not.

Here, on the other hand, we are dealing with a generic lattice, which has no definite order, and hence one cannot traverse the lattice from one side to the other in a regular way. Therefore it is not a priori clear which neighbours have already been visited and hence have been assigned a cluster label. This problem can be solved by a suitable initialisation. For instance, we can define arrays to hold the cluster label per site (LS(NSITES)) and per bond (LB(NBONDS)). During initialisation we can set LS (I) $=0$ for occupied sites and LS (I) $=-1$ for empty ones, and similarly for bonds. In the couse of the algorithm, the sites for which $\mathrm{LS}(\mathrm{I})=0$ will be assigned a valid label $\mathrm{LS}(\mathrm{I})=m$. Since valid labels are greater than zero, one can, at any time during the algorithm, recognise which sites are 'empty', which ones are 'occupied but unassigned', and which ones are 'assigned'.

Having performed the cluster algorithm, one still has to decide whether there is a percolating cluster or not. One has to define, therefore, what is percolation on this lattice: from where to where does a cluster have to extend, to be called percolating? In other words, one should define a beginning of the lattice and an end (or an 'IN' and an 'OUT'). For example, one can arrange the list of sites such that the last $N_{\text {out }}$ sites will be the exit of the lattice and the last $N_{\text {in }}$ before that will be the entrance. Having done so, one can loop over the entrance sites and mark the clusters that are connected to it. The final step is then to loop over the exit sites, to see whether there is a 'marked' cluster connected to the exit. If so, there is a percolating cluster, and otherwise there is not.

The marking of a cluster can be done in various ways, for example by adding $N_{s+b}$ to $N(m)$, where $N_{s+b}$ is the total number of bonds and sites. Because the total number of sites and bonds that can belong to any cluster is at maximum $N_{s+b}$, this method is allowed: any cluster with $N(m)>N_{s+b}$ is marked as connected to the entrance, and consists of $N(m)-N_{s+b}$ sites and bonds.

The cases of site-site percolation or bond-bond percolation are special cases of site-bond percolation. One can e.g. calculate the bond-bond percolation thresholds by simply making sure that all sites are 'occupied'.

The special purpose program to calculate the site percolation thresholds for several lattices is both faster and more economic in the use of memory, and was 
Table 4. The bond percolation thresholds of $d$-dimensional Kagomé, fcc and bcc lattices as a function of linear lattice size $L$. The values in the row marked with $\infty$ are results of a fit to the scaling relation $\left|p_{c}(L)-p_{c}(\infty)\right| \propto L^{-1 / \nu}$. Error estimates concerning the last digit are indicated between brackets. The values in the last row were obtained with an alternative method for extrapolation to $L=\infty$ (see text).

\begin{tabular}{rcccrccc}
\hline \multicolumn{4}{c}{$d=4$} & \multicolumn{5}{c}{$d=5$} \\
$L$ & Kagomé & fcc & bcc & $L$ & Kagomé & fcc & bcc \\
\hline 8 & $0.1725(2)$ & $0.0473(2)$ & $0.0729(2)$ & 6 & $0.1176(2)$ & $0.0227(2)$ & $0.0287(2)$ \\
12 & $0.1755(2)$ & $0.0484(2)$ & $0.0738(2)$ & 8 & $0.1237(2)$ & $0.0237(2)$ & $0.0301(2)$ \\
16 & $0.1764(2)$ & $0.0487(2)$ & $0.0738(2)$ & 10 & $0.1264(2)$ & $0.0252(2)$ & $0.0309(2)$ \\
24 & $0.1767(2)$ & $0.0490(2)$ & $0.0740(2)$ & 12 & $0.1275(2)$ & $0.0255(2)$ & $0.0314(2)$ \\
$\infty$ & $0.177(1)$ & $0.049(1)$ & $0.074(1)$ & $\infty$ & $0.130(2)$ & $0.026(2)$ & $0.033(1)$ \\
check & $0.179(2)$ & $0.049(1)$ & $0.075(1)$ & check & $0.132(2)$ & $0.027(1)$ & $0.033(1)$ \\
\hline
\end{tabular}

written in C. For the Bravais lattices, this is achieved by only using an array for the sites sites $(\mathrm{x}, \mathrm{y}, \ldots)$ and an array for $N(m)$. These lattices can be traversed in a structured way, so that we know in advance which neighbours have been visited before, and which ones haven't. The specification of the neighbours of a site is done, for the four-dimensional fcc lattice, by



These statements specify 12 neighbours, out of a total of 24 for a site on the fourdimensional fcc lattice. The first six of these neighbours correspond with the first line in Eq. (2), the last six with the second line. The above neighbours are only the ones that have been visited before, because the loop over sites is performed with the loop over ' $\mathrm{ia}$ ' as the outer-most loop, and the loop over ' $\mathrm{ix}$ ' as the inner-most loop. For each of the neighbours, ixp gives the x-displacement with respect to the current site.

For non-Bravais lattices, like the diamond lattice, the sites array was given an extra dimension $l$, where $l$ runs over the sites in the basis of the lattice. For the diamond lattice in $d$ dimensions, the basis consists of 2 sites. The loop over $l$ was implemented as the inner-most loop. The neighbours of a site can then be specified as follows. The sites of type A are treated differently from the sites of type B.

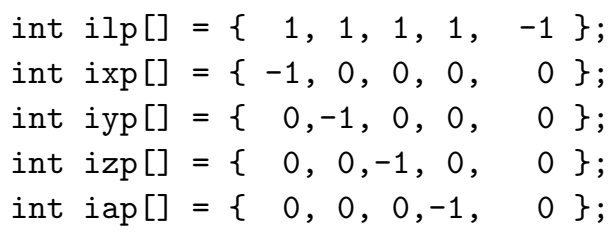

The first four neighbours are neighbours of the site of type A. They are sites of type $\mathrm{B}$, located at a displacement of $-\mathbf{x}$, respectively $-\mathbf{y}, \ldots$ The last neighbour is a neighbour of the site of type B. It is the other site, type A, in the same basis. 


\section{Results}

The percolation thresholds of fcc, bcc, and diamond lattices are given in Tables 1 4, for several lattice sizes. All the results were obtained with runs on a Sun Sparc workstation with $320 \mathrm{Mb}$ internal memory. The values in Table 1 were calculated by use of the general purpose program. This program allowed only limited lattice sizes, for which it is not sure that the scaling relation

$$
\left|p_{c}(L)-p_{c}(\infty)\right| \propto L^{-1 / \nu}
$$

holds. Therefore an estimate was made of a possible systematic error. This was done by comparing a fit of the scaling relation to the last three data points and a fit to the last two data points. The estimate is given as the error margin for $p_{c}(\infty)$ in the table. During the fitting procedure the value of the exponent $\nu$ was fixed at 0.88 in three dimensions, and $\nu=0.68,0.57,0.5$ in 4,5 and 6 dimensions respectively $t$.

As a check on the results for $p_{c}(\infty)$, we also used an alternative method to determine the value for the percolation thresholds at $L=\infty$. When we define

$$
\Delta(L)=\sqrt{\left|<p_{c}(L)^{2}>-<p_{c}(L)>^{2}\right|},
$$

we can use the relation

$$
\left|<p_{c}(L)>-p_{c}(\infty)\right| \propto \Delta(L) .
$$

With the use of this relation, the value of $p_{c}(\infty)$ can be fitted, without knowledge of the value of the exponent $\nu$. The results of this fit are shown in the row marked 'check' in Table 6 . They confirm the values of the row marked ' $p_{c}(\infty)$ '.

The results in Tables 13 were obtained using the special purpose program, which was designed to suit the particular lattice at hand. Fits to the data points show that the values in the tables are within the scaling regime, see Fig. 1. Therefore the error margins quoted here for $p_{c}(\infty)$ are the ones indicated by the fit. The values for the fcc lattices in Table 1 are different from those reported by Zallen 10.098 in $d=4$ and 0.054 in $d=5$ ). However, the cross-check with the general purpose program confirmed the numbers of Tables 1 3. Furthermore, it is not clear how the Zallen results have been calculated, nor how large their estimated error margin is.

\section{Discussion}

Percolation thresholds of many lattices are listed in Table 5. The results for the deviation of $C=\frac{\delta}{d} p_{c s}^{-1 / a_{s}} p_{c b}^{1 / a_{b}}$ from 1 is also listed. When we focus on the results for the invariant $C$, it is interesting to compare the triangular lattice with the octagonal lattice. Both lattices are isotropic lattices, with (average) coordination number 6 . Both lattices are fully triangulated, and hence have equal site percolation threshold $\$ p_{c s}=\frac{1}{2}$. Nevertheless, their bond percolation threshold differ: $p_{c p}=$ $2 \sin (\pi / 18)=0.347296 \ldots$ for the triangular lattice vs. $p_{c b}=0.3237 \pm 0.000610$ for the octagonal lattice. Therefore, the value of $C$ for these lattices is quite different, 


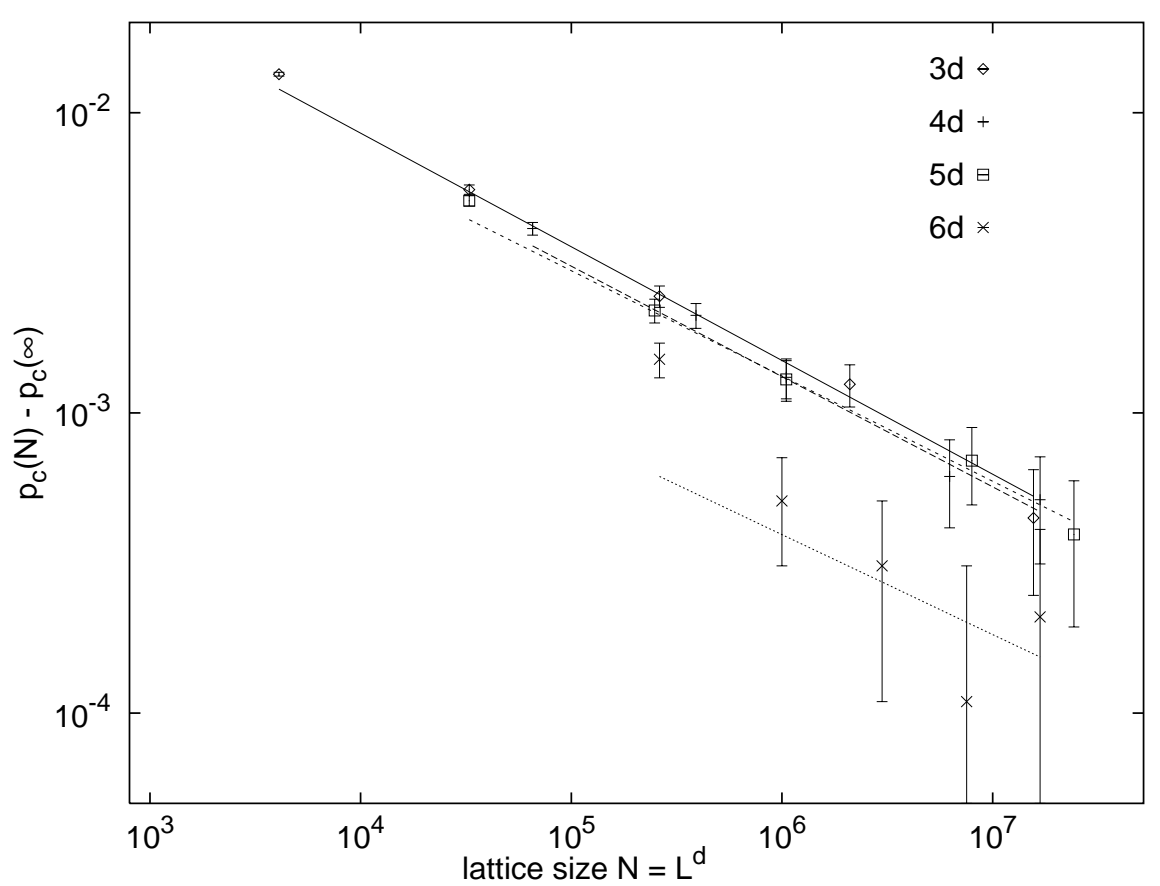

Fig. 1. The scaling of the bcc site percolation thresholds as a function of the lattice size see Eq. (4). The fits to the data have a slightly different slope, because the exponent $\nu$ is different for each dimension up to $d=6$.

namely $C=0.97$ for the triangular and $C=0.88$ for the octagonal lattice. Also for the dual of the octagonal lattice, the value for $C$ deviates substantially from unity: $C=0.91$.

One can also compare the cubic lattice and the 3-dimensional Kagomé lattice. The latter has a much higher site percolation threshold $(0.3895 \pm 0.0002$ vs. $0.311604 \pm 0.000000614$ ), but the bond percolation thresholds lie closer together $(0.2706 \pm 0.000912$ vs. $0.2488126 \pm 0.000000513)$. Although both lattices are isotropic and have coordination number 6 , the respective values for $C$ are 0.99 and 0.75 .

The dual of the diamond lattice and the 3-dimensional Kagomé lattice form a special pair too. It can be shown that the site percolation thresholds of these lattices are equal, although the lattices have a different coordination number 12 . On the other hand, their bond percolation thresholds are different, which is reflected in the values for $C$ being 0.75 and 0.65 . Note further that the dual of the bcc lattice, which was not incorporated in the Galam and Mauger study 3 , also shows a substantial deviation, with $C=0.89$.

Moreover, in high dimensions the dependence of percolation thresholds on the dimension $d$ is not universal. For the proposed invariant, Galam and Mauger assumed that all thresholds have equal scaling behaviour, such as the relation $p_{c} \propto 1 /(2 d-1)$ 


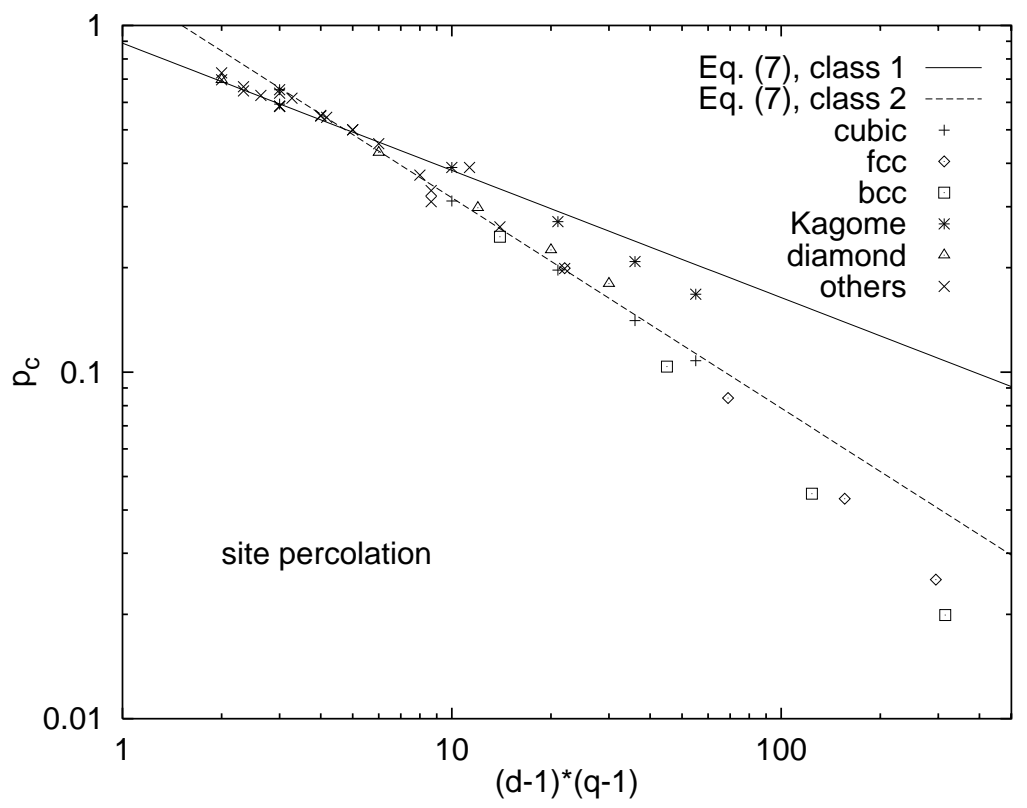

Fig. 2. Site percolation thresholds as a function of $(d-1)(q-1)$, see Eq. (7)

that was established some time ago for hypercubic lattices 4 . However, it has been shown recently 0 that there are $d$-dimensional Kagomé lattices with a different scaling behaviour, namely $p_{c} \propto 1 / d$. For these lattices the values for $C$ decrease with dimension, until $C=0.58$ for $d=5$. Also for the fcc, bcc, and diamond lattices, the behaviour as a function of dimension has not yet been captured fully. For each of the lattices the $C$-value deviates more strongly from unity, as the dimensionality increases. The scaling of percolation thresholds as a function of dimension was fitted by Galam and Mauger 3 to

$$
p_{c s} \propto\{(d-1)(q-1)\}^{-a_{s}}
$$

for site percolation thresholds, and

$$
p_{c b} \propto\left\{\frac{(d-1)(q-1)}{d}\right\}^{-a_{b}}
$$

for bond percolation thresholds. The thresholds have been plotted in Figs. 2 and 3 , together with the Galam and Mauger fit. The bond percolation thresholds, Fig. 3 , all seem to have the same trend, and the fit captures that trend quite well. The site percolation thresholds, however, show a much more complicated behaviour (Fig. 2). The deviations from the Galam and Mauger fit are increasing with dimension, but also it looks as if there is a spread in the thresholds, even though they follow a general trend. The Kagomé lattices should be considered separately, since their scaling behaviour has been shown to differ from the scaling for hypercubic lattices 


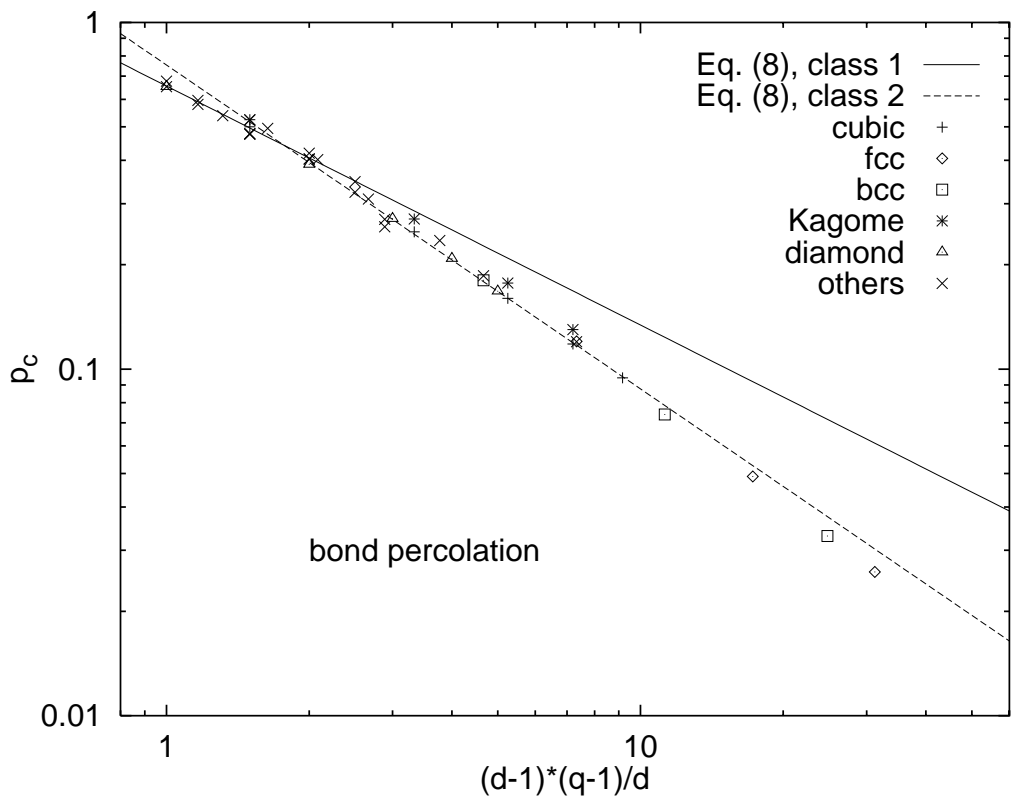

Fig. 3. Bond percolation thresholds as a function of $(d-1)(q-1) / d$, see Eq. (8)

In conclusion, site and bond percolation threshold have been calculated for several lattices in up to six dimensions. The scaling behaviour of these percolation thresholds as a function of dimension is sometimes different than for other known lattices. It may therefore be necessary to introduce more separate classes to allow for the variation in behaviour, e.g. a separate class for each dimension. If the deviations would then become smaller again, the invariant proposed by Galam and Mauger would still have the remarkable property of being independent of the coordination number.

\section{Acknowledgement}

The author would like to thank Bob Ziff for his help in compiling the table of percolation thresholds and the corresponding references.

\section{References}

1. D. Stauffer and A. Aharony, Introduction to Percolation Theory (Taylor and Francis, London, 1992).

2. S. Galam and A. Mauger, Phys. Rev. E53, 2177 (1996).

3. S. Galam and A. Mauger, Eur. Phys. J. B1, 255 (1998).

4. J.H. Conway and N.J.A. Sloane, Proc. R. Soc. Lond. A453, 2369 (1997).

See also http://www.research.att.com/ njas/lattices/

5. S.C. van der Marck, J. Phys. A: Math. Gen. 31, 3449 (1998).

6. J.H. Conway and N.J.A. Sloane, Discrete Comput. Geom. 13, 383 (1995).

7. J. Hoshen and R. Kopelman, Phys. Rev. B14 (1976) 3438. 
8. R. Zallen, The Physics of Amorphous Solids (John Wiley \& Sons, New York, 1983) p. 170.

9. M.F. Sykes and J.W. Essam, J. Math. Phys. 5, $1117(1964)\left(p_{\triangle}=2 \sin (\pi / 18)\right.$ is exact).

10. S.C. van der Marck, Phys. Rev. E55, 1514 (1997); Erratum E56, 3732 (1997).

11. P. Grassberger, J. Phys. A: Math. Gen. 15, 5867 (1992).

12. S.C. van der Marck, Phys. Rev. E55, 6593 (1997).

13. C.D. Lorenz and R.M. Ziff, Phys. Rev. E57, 230 (1998).

14. D.S. Gaunt, M.F. Sykes and H. Ruskin, J. Phys. A: Math. Gen. 9, 1899 (1976).

15. R.M. Ziff and B. Sapoval, J. Phys. A: Math. Gen. 19, L1169 (1986); R.M. Ziff, Phys. Rev. Lett. 69, 2670 (1992).

16. H. Kesten, Comm. Math. Phys. 74, 41 (1980) ( $p_{\diamond}=\frac{1}{2}$ is exact).

17. R.M. Ziff and P.N. Suding, J. Phys. A: Math. Gen. 30, 5351 (1997).

18. J.C. Wierman, J. Phys. A: Math. Gen. 17, 1525 (1984) ( $p_{\bowtie}$ is the root of $1-p-$ $\left.6 p^{2}+6 p^{3}-p^{5}=0\right)$.

19. F. Yonezawa, S. Sakamoto, and M. Hori, Phys. Rev. B40, 636 (1989).

20. F. Babalievski, Physica A220, 245 (1995).

21. F. Babalievski, cond-mat/9711164, subm. to Phys. Rev. E.

22. H.G. Ballesteros, L.A. Fernández, V. Martín-Mayor, A. Muñoz Sudupe, G. Parisi, and J.J. Ruiz-Lorenzo, Phys. Lett. B400, 346 (1997).

23. D.S. Gaunt and H. Ruskin, J. Phys. A: Math. Gen. 11, 1369 (1978).

24. S. Kirkpatrick, Phys. Rev. Lett. 36, 69 (1976). 
Table 5. Site $\left(p_{c s}\right)$ and bond $\left(p_{c b}\right)$ percolation thresholds for various lattices and their dual lattices. The deviation of the quantity $C=\delta p_{c s}^{-1 / a_{s}} p_{c b}^{1 / a_{b}} / d$ from 1 is listed under $C-1$. Between brackets are error estimates concerning the last digit. The thresholds without error margin are For the site percolation thresholds on cubic lattices in higher dimensions, see also Refs. $14,24$. The lattices in the upper part of the table are two-dimensional, including a number of aperiodic lattices; the ones in the middle are three-dimensional, and in the lower part of the table the higher dimensional lattices are listed.

\begin{tabular}{|c|c|c|c|c|c|c|}
\hline lattice & $p_{c s}$ & $p_{c b}$ & $C-1$ & $p_{c s}$ dual & $p_{c b}$ dual & $C-1$ \\
\hline square & 0.5927460 (5 & 0.516 & 0.04 & $\square$ & & \\
\hline Kagomé & 0.652703 .9 & $0.5244053(3)$ 너 & -0.02 & $0.5848(2) \stackrel{10}{=}$ & $0 . 4 7 5 5 9 4 7 6 3 \longdiv { 1 7 }$ & 0.00 \\
\hline pentagonal & $0.6471(6)$ 过 & $0.5800(6) 10$ & 0.01 & $0.5502(8)$ 目 & $0.4196(6) 10$ & -0.01 \\
\hline bowtie & 0.54760 & $0.404518 \ldots 18$ & -0.05 & $0.6653(6) 10$ & $0.595481 \ldots 18$ & -0.02 \\
\hline triangular & 0.52 & $0.347296 \mathrm{a}$ & -0.03 & $0.6971(2) \square$ & 0.652703 .9 & -0.02 \\
\hline octagonal & 0.5 & $0.3237(6) 10$ & -0.12 & $0.7297(4) 10$ & $0.6771(6) 10$ & -0.09 \\
\hline Penrose & $0.5837(3)$ 过 & $0.4770(1)$ & 0.01 & $0.6381(3) \longleftarrow 9$ & $0.5233(2) 19$ & 0.01 \\
\hline octag-chem & $0.585(1) 20$ & $0.478(3) 21$ & 0.01 & & & \\
\hline octag-ferr & $0.543(2)=20$ & $0.402(5) 21$ & -0.04 & & & \\
\hline dodec-chem & $0.628(2)$ & $0.538(1) 21$ & -0.01 & & & \\
\hline dodec-ferr & $0.617(3) 20$ & $0.4950(5)^{21}$ & 0.01 & & & \\
\hline cubic & $0.311604(-\mathrm{\rho} 11$ & 0.248812655 & -0.01 & & & \\
\hline 3d-Kagomé & $0.3895(2)$ & $0.2709(6) \frac{12}{1}$ & -0.25 & & & \\
\hline diamond & $0.4301(2)$ & $0.3893(2)$ 远 & -0.06 & $0.3895(2)$ 白 & $0.2350(5)$ & -0.35 \\
\hline trian. stack & $0.2623(2) \mathrm{a}$ & $0.1859(2) 10$ & -0.03 &  & $0.3093(2)$ 且 & -0.05 \\
\hline bcc & $0.2458(2) \mathrm{Q}$ & $0.1802875(10) 13$ & 0.04 & $0.4560(6) \cong$ & $0.4031(6)=$ & -0.11 \\
\hline fcc & $0.1994(2)$ 回 & 0.120163 尗10) & -0.05 & $0.3341(5) 2$ & $0.2703(3) 2$ & -0.03 \\
\hline hcp & $0.1990(2)$ & $0.1199(2) 10$ & -0.05 & $0.3101(5) 12$ & $0.2573(3) 12$ & 0.04 \\
\hline $4 \mathrm{~d}$-cubic & $0.196901(5) 22$ & $0.1600(2) 23$ & -0.01 & & & \\
\hline 4d-Kagomé & $0.2715(3)$ 目 & ח & -0.35 & & & \\
\hline 4d-diamond & $0.2978(2)$ & $0.2715(3) 5$ & -0.12 & & & \\
\hline fcc $\left(' D_{4}\right.$ ') & $0.0842(3)$ & $0.049(1)$ & 0.13 & & & \\
\hline $4 \mathrm{~d}-\mathrm{bcc}$ & $0.1037(3)$ & $0.074(1)$ & 0.25 & & & \\
\hline 5d-cubic & $0.1407(3)$ 具 & $0.1181(2) 23$ & -0.04 & & & \\
\hline 5d-Kagomé & $0.2084(4)$ 目 & $0.130(2)$ & -0.42 & & & \\
\hline 5d-diamond & $0.2252(3)$ & $0.2084(4) 5$ & -0.16 & & & \\
\hline fcc $\left(' D_{5}\right.$ ') & $0.0431(3)$ & $0.026(2)$ & 0.39 & & & \\
\hline $5 d-b c c$ & $0.0446(4)$ & $0.033(1)$ & 0.69 & & & \\
\hline 6d-cubic & $0.1079(5)$ 具 & $0.0943(2) 23$ & 0.01 & & & \\
\hline 6d-Kagomé & $0.1677(7)$ 目 & & & & & \\
\hline 6d-diamond & $0.1799(5)$ & $0.1677(7) 5$ & -0.19 & & & \\
\hline fcc $\left({ }^{\prime} D_{6}\right.$ ') & $0.0252(5)$ & & & & & \\
\hline $6 d-b c c$ & $0.0199(5)$ & & & & & \\
\hline
\end{tabular}

